\title{
भारतीय ग्रामीण परिवेश में विकास के परिप्रेक्ष्य में ऋण के अर्थशासत्र की समझः
}

\section{समीक्षात्मक अवलोकन}

\section{डॉ. लोकेश जैन}

\section{सारांश-}

भारतीय अर्थव्यवस्था का एक बड़ा भाग कृषि पर आधारित है। 20 वीं सदी के आरंभ में सहकारिताओं के जरिये आर्थिक मदद का एक कदम आगे बढ़ा। उस समय इस लोक आधारित सहकारी संगठन का मुख्य ध्येय ऋण प्रदान करके किसानों की मुश्किलों को कम करना था। जरूरतमंद वर्ग, मजदूर, किसान, मध्यम वर्ग सभी को अपनी बुनियादी व विकासात्मक जरुरतें पूरी करने हेतु आर्थिक मदद की आवश्यकता रहती थी जिसके लिए वे स्थानीय शाहूकारों के पास उनकी ही शर्तों पर ऋण लेने को मजबूर थे। गांधीजी ने संयममय जीवन के अभिगम को अपनाने की आवश्यकता पर इसीलिए बल दिया एक तरफ इससे अतिरेक उत्पादन में लगने वाले साधन-संसाधनों का विनाश होने से बचेगा, पर्यावरण संतुलित रह सकेगा तथा लोगों रात-दिन कमाने तथा ऋण भुगतान करने की तानाव से मुक्ति मिल सकेगी जो उन्हें शांति व सुखमय जीवन जीने के लिए प्रेरित करेगी क्योंकि ऋण ऐसी प्यास है कि जो बुझने का नाम ही नहीं लेती, अवास्तविक सुख की ओर आकर्षित कर अंततः जोखिम की तरफ ढकेल देती है।

\section{ॠण और विकास का एतिहासिक परिप्रेक्ष्य....}

भारतीय अर्थव्यवस्था का एक बड़ा भाग कृषि पर आधारित है। कृषि क्षेत्र में विविध प्रकार की सामाजिकआर्थिक असमानाताएं विद्यमान हैं। वर्षा आधारित कृषि में अनिश्चितताओं की भरमार है तथापि भारतीय किसान व समाज उसके अनुरूप स्वयं ढालता रहा है और नियति के अनुसार स्वाभाविक रूप से जीवनयापन करता रहै है। समाज की इस कड़वी सच्चाई को नहीं नकारा जा सकता कि सदियों से सामाजिक आर्थिक वर्गभेद की खाई यहाँ विद्यमान रही है। जिसमें मुठ्ठीभर अमीर और ढेरों गरीब शामिल हैं। अमीरों का महत्तम साधन संसाधनो पर कब्जा भी रहा है। (इन छोटे, सीमान्त व जरूरतमंद लोगों का प्रतिशत मुठ्ठीभर उन सम्पन्न व धनाढ्य लोगों की तुलना में काफी अधिक है जो संबंधित उत्पादन संसाधनों के एक बड़े भाग पर काबिज हैं और मालिकाना हक रखते हैं।) यही शोषित वर्ग है जिसे अपनी 


\section{Jain / Page 50-65}

जरूरतों को पूरा करने के लिए मंहगी ब्याज दरों पर ऋण लेने के लिए विवश होना पड़ता था। आजादी से पूर्व उन्नीसवी-बीसवीं सदी में इस व्यवस्था के अध्ययन के लिए कई आयोग बैठाए गए जिन्होंने इन्हें शाहूकारी चुंगल से मुक्त कराने हेतु सुद्धढ़ ऋण व्यवस्था की स्थापना की सिफारिशें की। भारतीय सहकारिता अधिनियम,1904 के मूल में किए गए अध्ययन यह बताते हैं कि किसान अपनी जमीन के मालिक नहीं रह पा रहे थे। दुष्कालों के कारण पैदावार नहीं हुई और किसानों को अपनी जरूरतें पूरा करने के लिए शाहूकारों से $36 \%$ तक के उच्च ब्याज दरों पर ऋण लेने पर विवश होना पड़ा। उनकी जमीने सस्ते में बिना मोल के हड़प लेने का षडयंत्र अपने पुर जोर पर था।

ग्रामीण जनसंख्या का एक बड़ा भाग ऐसा रहा है जो अपनी उत्पादकीय व उपभोग संबंधी जरूतों को पूरा करने के लिए पर्याप्त अर्थोपार्जन नहीं कर पा रहा है। आय के स्रोत कितने ही मर्यादित क्यों न हों किन्तु जीवन-यापन हेतु, बुनियादी दैनिक व प्रासंगिक जरूरतों को पूरा करने के लिए, सामाजिक-सांस्कृतिक प्रथाओं न्यूनतम मापदण्डों पर भी निभाने के लिए, सामाजिक- पारिवारिक जिम्मेदारियों को पूरा करने, शिक्षण के क्षेत्र में विकास करने, परिवार का आरोग्य सुनिश्चित करने, व्यक्तिगत शारीरिक-मानसिक विकारों की संतुष्टि करने, व्यावसायिक एवं उत्पादकीय आर्थिक जरुरतों को पूरा करने तथा जीवन स्तर को बेहतर बनाने आदि के लिए उन्हें वर्तमान आय के अतिरिक्त आर्थिक मदद की आवश्यकता महसूस होती रही है। इस आर्थिक मदद को ऋण के रूप में जाना जाता है।

भारत में एक लम्बे समय तक ऋण का कारोबार असंगठित क्षेत्र सेठ, शाहूकार, बनिया आदि के हाथों में रहा। जिन्होंने बड़ी ब्याज दरों पर, ब्याज पर ब्याज रखकर अपना उधार दिया हुआ धन वसूलने में कोई कोर-कसर नहीं छोड़ रखी थी। शोषण की तो पराकाष्ठा ही थी। ब्याज का यह गोरख धंधा इतना बेइमानी से भरा था कि एक बार दिया हुआ कर्ज खत्म होने का नाम ही नहीं लेता था। एक बार कर्ज लेने वाला उस शाहूकार का जड़खरीद गुलाम जैसा बन जाता था और उस ऋण को चुकाने के लिए वही नहीं अपितु उसकी पीढ़ियां भी बाध्य होती थीं किन्तु वह फिर भी पूरा नहीं होता था। शायद इसीलिए यह लोकोक्ति चलन में आयी होगी कि भारतीय किसान कर्ज में ही जन्म लेता है और कर्ज में ही मर जाता है। यहाँ तक कि वह विरासत में अपनी संतान के लिए कर्ज की ही सौगात छोड़कर जाता है। कर्ज वसूली इस कदर निष्ठुर व निर्दयी थी कि कई किस्सों में तो मानवता भी शर्मसार होकर मृतप्राय बन जाती थी जिसके वर्णन में शब्द भी कम पड़ने लगते है। कोई भी अकाल, बाढ़, महामारी आदि कुदरती आपत्तियों में भले ही इनकी फसलें आदि आजीविका स्रोत क्यों न गिरकर तहस नहस हो गये हों किन्तु इऩ शाहूकार का कर्ज पठान जैसी वसूली के लिए खड़ा ही रहता था।

\section{ग्रामीण क्षेत्र में संगठित ऋण व्यवस्था के आयाम-}


ऐसे में 20 वीं सदी के आरंभ में सहकारिताओं के जरिये आर्थिक मदद का एक कदम आगे बढ़ा। उस समय इस लोक आधारित सहकारी संगठन का मुख्य ध्येय ऋण प्रदान करके किसानों की मुश्किलों को कम करना था। इनका जोर भी उत्पादकीय ऋण प्रदान करने पर था। ये संगठित क्षेत्र उतने सुदृढ़ होकर नहीं उभरे थे जितनी लोगों की जरूरतें थी फिर ये लोगों की मर्यादित क्षेत्र की जरूरतों को पूरा करने के लिए ऋण जुटा पाते थे और शेष अनुउत्पादकीय किन्तु जीवन की आवश्यक व्यक्तिगत-सामाजिक जरूरतों के लिए फिर उन्हें इन्हीं शोषित करने वाले महाजनी स्रोतों पर आश्रित रहना पड़ता था। हालांकि 1935 में भारतीय रिजर्व बैंक के पदार्पण के पश्चात एक ओर बैंकिंग क्षेत्र इस दिशा में सक्रिय हुआ जिसका इस व्यवस्था में हस्तक्षेप एक सीमा तक महसूस किया जा सकता था। किन्तु इनके कार्य मर्यादित थे, औपचारिकताऐं इतनी अधिक थी कि लोग दूर से ही हाथ जोड़ लेते थे। वसूली के मापदण्ड बहुत कड़क थे। हां शाहूकारों के किस्से में इतनी बात अवश्य थी कि यदि काश्तकार की जमानत स्वरूप रखी सम्पत्ति में कोई गुंजाइश होती थी तो वसूली का समय कुछ समय के लिए आगे खिसक जाने की संभावनाएं बनी रहती थी वो तुंरत उस आसामी की जायदाद को नीलाम नहीं कराते थे जो उसके जीवन का आधार थी। जबकि बैंक के किस्से में यह कानूनी तौर पर होता था जिससे किसान में डर सा बैठ गया। 70 के दशक में बैंको का राष्ट्रीयकरण होने के पश्चात इस क्षेत्र की एवं सरकारी हस्तक्षेप की असरकारकता का दायरा बढ़ा। कम मुनाफा होने के बाबजूद सरकार की विकास संबंधी प्राथमिकताओं के चलते लीड बैंक व क्षेत्रीय ग्रामीण बैंको की शाखाओं का विस्तार गावों में हुआ, सहकारिताओं की भी दशा सुधरी, कार्य क्षेत्र में काफी विस्तार हुआ अर्थात कई क्षेत्रों में साख सुलभ होने लगी फिर भी वे सभी मिलकर लोगों की जरूरतों को पूरा करने का सुद्ढ़ विकल्प नहीं बन सकीं। कई प्रकार की बाधाऐं थी आड़े जिनके चलते लोग इनका उपयोग जरूरतों को पूरा करने, जीवन स्तर को ऊपर उठाने तथा सामाजिक-शैक्षणिक क्षेत्र में विकासलक्षी प्रवृत्तियों का लाभ नहीं लेने में नहीं कर पा रहे थे।

90 के दशक में आर्थिक उदारीकरण के पश्चात फिर से नये क्षितिज खुले। निजी वित्तीय संगठन इस क्षेत्र में आगे आए। किन्तु विश्वसनीयता की कमी अभी भी खल रही थी। ये संस्थाएं कब तक चलेंगी, कब तक अपने बनाए नियमों पर जमी रह सकेंगी, आमआदमी तय नहीं कर पा रहा था। इनका छद्म ऐजेन्डा क्या है कुछ भी स्पष्ट तौर पर नहीं कहा जा सकता था। कई सारी वित्तीय संस्थाएं भाग बाजार छोड़कर भाग खड़ी हुईं। कुछ भी हो फिर भी इन्होंने शाहूकारों की एकाधिकार की व्यवस्था पर करारा वार किया। इस दौरान विकास के प्रतिमान भी बदले। स्वयं सहायता समूहों का चलन 21 वीं सदी के आरंभ में हो गया था। मतलब यह कि जरूरतमंद जिनके पास मर्यादित साधन थे, जमानत राशि का अभाव था, उत्पादकीय कार्यों को संचालित करने की आर्थिक क्षमता नहीं थी उनके लिए वित बजार में इस निजी क्षेत्र के जरिए तथा स्वैच्छिक संगठनों, सरकारी व अर्धसरकारी क्षेत्र के सौजन्य से ऋण क्षमतावृद्धि, आजीविका विकास व जरूरत संतुष्टि की संभावनाएं पैदा हुई। लोगों की ऋण लेने व भुगतान अर्थात ऋण 


\section{Jain / Page 50-65}

वापसी की क्षमता भी बढ़ी और वे समूह अपनी सामाजिक व बिन उत्पादकीय आवश्यकताओं की पूर्ति करने में भी एक सीमा तक स्वयं को अथवा समूह को समर्थ महसूस करने लगे। लगभग गत डेढ़ दशक से इस ऋण क्षेत्र में आमूलचूल परिवर्तन आये हैं जिसने उत्पादक क्षेत्र ही नहीं अपितु बुनियादी जरूरतों के प्रतीक आवास, मंहगी व टिकाउ परिसम्पत्तियां, गोल्ड, वाहन, घरेलू सामान आदि के लिए ऋण सुविधाओं का बजार बढ़ा जिसके परिणामों ने आज यह विश्लेषण करने को विविश कर दिया है।

\section{ऋण का मकसद तब और अब- प्रदान करने व लेने वाले की दृष्टि में....}

तब....

जरूरतमंद वर्ग, मजदूर, किसान, मध्यम वर्ग सभी को अपनी बुनियादी व विकासात्मक जरूरतें पूरी करने हेतु आर्थिक मदद की आवश्यकता रहती थी जिसके लिए वे स्थानीय शाहूकारों के पास उनकी ही शर्तों पर ॠण लेने को मजबूर थे। यदि उनके पास जड़ जायदाद होती थी तो उसकी तरफ उनकी आँख रहती थी और वे येनकेन प्रकारेण इन किसानों को सुनियोजित रूप से अपनी हड़प नीति का शिकार बना लेते थे और उनको दासता के लिए मजबूर करते थे, उनसे करने व न करने वाले सभी कामों को बलात् कराते थे। कई बार तो इन शाहूकारों का व्यवहार मीठी छुरी की भाँति होता था अर्थात उसे अधिक से अधिक बिन जरूरी ऋण लेने के लिए प्रोत्साहित करते थे और न चुका पाने की स्थिति आने पर अपनी हड़प नीति को अंजाम दे देते थे और फिर इन्हें अपने व्यवसाय से दूध में से मक्खी की तरह निकाल कर फेंक दिया करते थे। तब ये ऋण लेने वाले अपने आप को असहाय ही पाते थे क्योंकि इनकी कोई सुनने वाला और उन पर विश्वास करने वाला कोई नहीं होता था। पुराना ऋण चुकाने के लिए ब्याज पर ब्याज लगाकर नया ऋण भी प्रदान कर दिया करते थे। काश्तकार इस दुष्चक्र को इनकी महरबानी मानकर आनन्द के भ्रम में जीता था अथवा जानने-समझने की स्थिति में मन मसोस कर यह सब करने को मजबूर होता था। ऋण की इस कुव्यवस्था में पिसने के अतिरिक्त उसे कुछ भी हासिल नहीं होता था सिवाय पश्चाताप के। "जब चिड़िया चुंग गई खेत, तब पछताए होत क्या" यह लोकोक्ति ही इन शोषितों पर लागू होती थी।

लेखक ने उत्तर प्रदेश, राजस्थान व गुजरात राज्य के ग्रामीण व आदिवासी विस्तार में किए गए विभिन्न शोध अध्ययनों एवं लोगों के साथ किए गए साक्षात्कारों में पाया कि ग्रामीण विस्तार में गरीबों की लगभग विकल्प रहित इसी मजबूरी का फायदा उठाकर महाजन समुदाय इन काश्तकारों, मजदूर वर्ग एवं भूमिहारों का अनेकानेक रूप से शोषण किया करता था जैसे- अधिक ब्याज दर, ब्याज पर ब्याज लगाना, ब्याज की राशि अग्रिम काटकर ऋण देना, उनके उत्पादों को सस्ते में खरीदने तथा अपने माल-सामान के मंहगे में खरीदने का दबाब बनाना, उनसे एवं उनके परिवार से बेगार लेना अर्थात् उनके द्वारा दी गई 
सेवाओं का कोई आर्थिक मूल्य नहीं गिनना आदि ये तो मात्र आर्थिक शोषण के ही उदाहरण है किन्तु इससे आगे उनके सामाजिक मामलों में अनुचित दखलंदाजी करना, शारीरिक-मानसिक शोषण का शिकार बनाना, कुदरती आपत्ति होने पर भी वसूली में अमानवीय तरीकों को अपनाना, जलील करना, थोड़ा थोड़ा चुकाते रहने पर भी कर्जे का ज्यों का त्यों खड़े रहना और यह कहना कि वह तो तुम्हारे ब्याज मे जमा हुआ है, अनुचित व्यापार व्यवहार अपनाना अर्थात् लिखापढ़ी में फेरफार कर देना आदि ढेरों उदाहरण दृष्टिगत होते हैं। चुकाते चुकाते भी कर्जे का उतना ही बना रहना, कई बार व्याज का मूलधन से कईगुना अधिक हो जाना भी सामान्य बात है। यह कर्ज पीढ़ी दर पीढ़ी चलता रहता है। कई किस्सों में इन परिवार के बच्चों को पूर्णरूप से ज्ञात ही नहीं होता कि उनके पिताजी ने वास्तव में कितना कर्ज किस रूप में किस कार्य के लिए लिया था और किन लिखित-मौखिक शर्तों पर। लेकिन उन्हें समाज में रहते हुए इस तथाकथित ऋण को चुकाने के बाध्य होना होता है।

अब.....

ॠणग्रस्तता की स्थिति पर आजादी से पूर्व भी सरकारों का ध्यान गया तथा आजादी के बाद पंचवर्षीय योजनाओं के जरिए एवं बीस सूत्रीय कार्यक्रम आदि के तहत गंभीरता से विचार किया गाया। शाहूकारों की स्थापित व्यवस्था के सामने संगठित क्षेत्र के रूप में बैंकिंग क्षेत्र को उतारा गया। इस ऋण प्रदान करने वाली संस्थागत संरचना का मुख्य ध्येय लोगों की वित्तीय समस्याओं का समुचित समाधान प्रस्तुत करना था। किन्तु ये संस्थाएं विशुद्ध व्यावसायिक संस्थाओं के रूप में उभरीं इसलिए सुरक्षित ऋणों व नफाकारकता को केन्द्र में रखकर इनकी गतिविधियां संचालित होती रहीं। शहर व उद्योग जगत एवं वे लोग जिनके पास जमानत राशि की सुगमता थी, इससे लाभान्वित हुए। शायद सरकारें भी कमोवेश प्रसन्न थीं कि इस तरह से जीडीपी में वृद्धि तो हो रही थी। किन्तु कुछ समय पश्चात देश के कर्णधारों का ध्यान जरूरतमंदों की ऋणग्रस्तता की ओर गया। बैंको के राष्ट्रीयकरण का निर्णय लिया गया, ग्रामीण बैंक व क्षेत्रीय ग्रामीण बैंकों की कल्पना को आकार दिया गया ताकि वे गांवो तक पहुँच कर किसनों व जरूरतमंदों की वित्तीय सहायता कर कृषिगत एवं कृषि संबंधित क्षेत्र को उन्नत बना सकें और उन्हे शाहूकारी ऋणग्रस्तता से उबार सकें। काश्तकारों व भूमिहारों से साक्षात्कार करते हुए ज्ञात हुआ कि बैंकों में कागजी कार्यवाही की अधिकता के कारण उनमें से अधिकांश इस मकसद के लिए बैंक जाना पसंद नहीं करते थे। वास्तविकता यही थी बैंक छोटे छोटे ऋणों में रूचि नहीं रखते थे, गरीबों के साथ उनका व्यवहार साहिबी वाला होता था इसलिए शोषित होते रहने पर भी वे शाहूकार के पास जाना ही अधिक पसंद करते थे। दूसरा एक अन्य कारण था कि ऋण मिलने में देरी जबकि उसे ऋण की त्वरित जरूरत पड़ती थी रात हो दिन उस जरूरत के मद्देनजर समय का इंतजार करना संभव नहीं था लेकिन बैंक तो अपने कार्य-समय में ही कुछ सकते थे। जिंदगी में सभी जरूरतें तो पूर्व नियोजित होती नहीं हैं इसलिए इस जीवनशेली में वे चाहकर भी कुछ नहीं पाते थे। 
शाहूकारों के दुष्चक्र से मुक्ति दिलाने के लिए बैंक जैसे जो संगठित वित्तीय संस्थान आगे आये उन्हें ऋण तो प्रदान करना था किन्तु एन.पी.ए. को अंकुशित रखने एवं नफाकारकता के वांकित दर को बनाए रखन हेतु ऋण वापसी की पूर्ण सुनिश्चितता भी करनी थी इसलिए प्रक्रिया व शर्तों को थोड़ा जटिल व संरक्षित बनाया गया वसूली को भी कड़क रखा गया। यहाँ तक कि शाहूकार के किस्से में यदि उसे दया आ जाय तो उसकी वसूली को मुल्तवी कर दिया जाता था जबकि बैंक तो इसमें एक कदम आगे ही थे वे तो कानून का डर दिखाकर उसकी ही आड़ लेकर पठान की तरह वसूली करते थे। छोटे व जरूरतमंदो को निजात कहाँ थी इस व्यवस्था में उन्हें या तो सिक्युरटी के रूप में संपत्ति को बताना होता था या किसी ऐसे आदमी को जमानत देने के लिए तैयार करना होता था जिसकी उतनी आर्थिक साख हो कि उसे बैंक से ऋण मिल सके। यदि इसकी पहली व्यवस्था की बात करें तो यह साफ था उस जरूरतमंद के पास मिल्कत का अभाव था अथवा सिक्युरटी में देने के लिए पर्याप्त नहीं था इसलिए उसे ऋण मिलना मुश्किल था। दूसरा विकल्प अपनाने पर उसे कई प्रत्यक्ष और परोक्ष मुश्किलों का सामना करना पड़ता था। यथा पहले तो कोई भी सम्पन्न व्यक्ति उस गरीब जरूरतमंद की जमानत को तैयार होने के पचड़े में पड़ना ही नहीं चाहता था और यदि दया खाकर तैयार हो भी गया तो बार बार अहसान जताकर, नौतिकता का भय दिखाकर उसे और उसके परिवार को बेगार देने के लिए मजबूर करता और नानाविध शोषण करता रहता था अन्यथा जलील करता था। दूसरे बैंक का कार्य समय दिन में 10-05 होता है उसे इसी सम दरम्यान ऋण के लिए आवेदन करना होता है, औपचारिकताएं पूरी करनी होती हैं, तब कहीं जाकर ऋण मिलता है लेकिन उसकी आकस्मिक जरुरतें तो रात समाप्त होने इंतजार नहीं करती और न ही छुट्टी के दिन की बाधा को मानती हैं ऐसे में उसे स्थानीय शाहूकारों की शरण होना पड़ता था और उन्हीं की शर्तों पर। बैंक में जाने पर तो शाहूकारों के साथ आर्थिक व्यवहारों के संबंध और अधिक खट्टे तथा कड़क हो जाते हैं। मतलब यह कि छोटा और गरीब आदमी अपनी ऋण की जरूरतों को पूरा करने के लिए बैंक जाने के बजाय स्थानीय स्रोतों पर जाकर जररतें पूरी करने में ही अपनी भलाई समझने लगा, यह रास्ता उसे ज्यादा सरल लगा। हाँ बैंकिंग सिस्टम के आने से इन शोषणखोर, ब्याजखोर शाहूकारों की कई प्रकार की ज्यादातियों पर रोक अवश्य लगी। दूसरे वातावरण में शिक्षण का प्रभाव दिखा किसानों की भुगतान वापसी की क्षमता भी बढ़ी उनके बच्चे बेहतर आजीविका हेतु शहरों की ओर स्थानान्तरित हुए, नौकरी आदि में भी लग गए इसलिए यह प्रभाव कम हुआ।

90 के दशक में उदारीकरण की नीति एवं विकास कार्यक्रमों के बदले अंदाज के चलते ऋण प्रदन करने की यात्रा भी उदारता की ओर बढ़ी। किसानों की कर्जमाफी का राजनैतिक खेल चला। यद्यपि मजबूत किसानों ने इसका सबसे अधिक लाभ उठाया। हालांकि समन्वित ग्रामीण विकास कार्यक्रम, महिला सशक्तिकरण, कौशल्य विकास आदि के माध्यम से उत्पादक ऋण सुविधाएं छोटे व जरुरतमंद लोगों तक पहुँची जिन्होंने उनके लिए उद्यमिता के द्वार खोले। तथापि कुछ तथ्य ऐसे उजागर हुए जो उनकी इस 


\section{Jain / Page 50-65}

संगठित क्षेत्र के प्रति उदासीनता को जाहिर करते हैं। जैसे सरकार ऋण की राशि जाहिर करती थी किन्तु उसे सामान के रूप में ही उनकी ही निर्धारित दुकान से लेना होता था। उनका आरोप था कि इसमें माल सही गुणवत्ता व उचित किंमत वाला नहीं होता था। बैंक के अधिकारियों से बातचीत करने पर ज्ञात हुआ कि यह सरकार की नीति थी जिस पर वे मात्र अमल करते थे। सरकार के ऐसा इसलिए किया क्योंकि लोग ऋण तो ले लेते थे किन्तु व्यवसाय के लिए उसका उपयोग न करके अन्यत्र उपयोग कर लिया करते थे जिससे न तो ऋण का मकसद पूरा होता था और न ही बैंको को ऋण वापिसी की सुनिश्चितता होती थी। फिर भी इस मायाजाल में अधिकारियों के कमीशन के रूप में बहुत घोटाला किया। जब चालाक लोगों को यह खेल पता चला तो उन्होंने भी बहती नदी में हाथ धोने से कोई गुरेज नहीं किया। कागजों में ऋण भी लिया गया, सामान भी नहीं खरीदा गया और सरकारी सब्सीडी का भी बँटबारा भी बदस्तूर हो गया। कर्जमाफी की गन्दी राजनीति की आड़ में तथाकथित जागृत ग्रामीण समुदाय ने बड़े बड़े ऋण लिए और भगतान भी नहीं किया बैंक भी सरकारी निबटारे की प्रक्रिया के आदी बनते गए।

उदारीकरण, निजीकरण और वैश्वीकरण के दौर में बैंक, व्यवसाय और विकास की प्राथमिकताएं संयुक्त रूप से सामने आयीं। यथा किसानों को कृषि हेतु खाद, बीज, दवा, छोटे बड़े यंत्र साधन आदि आदान उपलब्ध कारना, सिचाई की सुविधा को बेहतर बनाने के ड्रिप व अन्य योजनाओं को अमली बनाना जिसमें समुदाय विशेष की, क्षेत्र विशेष की स्थिति के अनुसार सरकारी सहायता के प्रावधान थे। ट्रेक्टर्स आदि के लिए बड़े ऋण भी उपलब्ध कराए गए। इसकी उत्पादन, वितरण, संग्रहण और मार्केटिंग व्यवस्था से जुड़े सभी स्टेकहोल्डर्स की रूचि सघनतम होती गई। ग्रामीण जनजीवन एवं वास्तविक लक्ष्य समूह पर इसका दीर्घगामी असर क्या हुआ इसकी चर्चा अगले भाग में करेंगे। अभी हम आज ऋण सुविधा प्रदान करने वाले संगठित क्षेत्र की मंशा, दशा और दिशा का साक्षात्कार कर लें।

आज हमारे देश में ऋण सुविधाओं का जाल बिछा हुआ है, सरकारी, निजी व सहकारी आदि सभी प्रकार के उपक्रम इससे जुड़े हुए हैं। एक नजर से देखने पर महसूस होता है कि ऋण के नाम पर जो सोच शाहूकारों की होती थी कमोवेश वही नजर यहाँ इस संगठित क्षेत्र में बनती जा रही है। ये संगठित क्षेत्र ॠण समस्याओं के समाधान की आड़ में, वास्तविक जरूरतों की संतुष्टि के स्थान पर जरूरतें खड़ी करने की धुन में उपभोग स्तर को बढ़ाने हेतु उदार ऋणनीति प्रारंभ की ताकि वे अधिक से अधिक नफा कमा सके भले ही इससे लोगों की जीवनशेली अस्तव्यस्त हो अथवा उसका स्वास्थ्य बिगड़े। इसके लिए वे लुभावने, आकर्षक प्रस्तावों के साथ लोगों को विविध प्रकार की ऋण सुविधा उपलब्ध कराने को लालायित हो रहे हैं यथा- क्रेडिट कार्ड, शून्य प्रतिशत प्रोसेसिंग फी, आसान किस्तें। इससे समाज में आर्थिकविपन्नता, तनाव, अवसाद आदि का केंसर तेजी से अपने पैर फेलाता जा रहा है। अब व्यक्ति को आने वाली आय, पगार की खुशी नहीं होती अपितु पहले से व्यय हो जाने से अभावसा अवश्य महसूस होता है। इसी वजह से अधिकांश लोगों की आय खाए हुए खेत की तरह हो चली है। ऋण फैला रहा है 


\section{Jain / Page 50-65}

किश्तों का केंसर। कई बार निर्णय बजार से सम्मोहित होकर ले तो लिए जाते हैं किन्तु उसके संचालकीय व्ययों को पूरा करने हेतु कुछ लोग तो अपराधिक प्रवृत्तियों की ओर भी उन्मुख हो जाते हैं। बैंक जो ऋण के द्वारा लोक-मदद के लिए आगे हाथ बड़ा रहे थे, उन्होंने ऋण को ही अपना व्यवसाय बना लिया अधिकतम ऋण का टर्नओवर ही प्रगति की पहचान बन गई। ऋण के विविध उपयोग और उसके प्रभावों पर ध्यान नहीं दिया गया। जरूरत आधारित होने की बजाय बजार आधारित होते चले गए हम। जरूरतें पूरी करने के बजाय जरूरतें खड़ी करके अपना उल्लू सीधा करने में लग गए। उदारीकरण और नोट बंधी के बाद तो इस व्यवस्था में आमूलचूल परिवर्तन आए। इसने एक मुहिम का रूप ले लिया। बड़े कर्जादारो- विजय माल्या जैसों को आसानी से माफी मिल जाती है तथा छोटों को तो आत्महत्या को लिए मजबूर होना पड़ता है, विभिन्न प्रकार की धमकियों का सामना करना पड़ता है। नोटबंदी और डिजिटलाइजेशन की अनिवार्यता की सरकारी नीति ने तो बैंक-ग्राहक संबंधो के स्वरूप को जड़ल से बदलकर रख दिया है। बैंक उनके हर ट्रांजेक्सन पर मनमाना शुल्क वसूल कर रहे है ये संबंध मालिकगुलाम जैसे बन गए हैं क्योंकि उन्हें ज्ञात है कि अब आम आदमी अथवा ग्राहक के पास उनकी तरह से ही कटने के अलावा कोई चारा नहीं बचा है।

ॠणजनित गुलामी बहुत बुरी होती है। ऋण लेने वाले को चाहे-अनचाहे समझौते करने ही पड़ते हैं। अन्तर्राष्ट्रीय पटल पर देखें तो अभी हाल ही में श्रीलंका जैसे देश को चीन को अपना व्यापारिक दृष्टि से महत्ववपूर्ण बंदरगाह देना पड़ा। हमें भी विश्वबैंक के प्रत्यक्ष और परोक्ष दबाब में कई ऐसे समझौते करने पड़े हैं जो हमारी अर्थव्यवस्था, समाज व्यवस्था एवं संस्कृति के अनुकूल नहीं थे।

हम सभी इस बात से भलीभांति परिचित हैं कि संगठित रूप से ऋण सुविधाओं का विकास हमारी विकास प्राथमिकताओं में शोषणमुक्ति, गरीबी, बेरोजगारी निवारण, औद्योगिक एवं व्यावसायिक उद्यमिता का विकास, जीवन की बुनियादी आवश्यकताओं की संतुष्टि, आकस्मिक किन्तु अनिवार्य आवश्यकताओं की संतुष्टि, बेहतर जीवन यापन की सुनिश्चितता आदि का केन्द्रीय स्थान हुआ करता था अथवा इसको ध्यान में रखकर चलना उनको लिए ध्येय वाक्य जैसा था। किन्तु हम देख पा रहे हैं कि बैंको व वित्तीय संस्थान भी विकृत हो चली शाहूकारी व्यवस्था का ही पर्याय बनकर रह गए हैं, करनी कुछ और है तथा दिखावा कुछ और। जीवन स्तर में सुधार के नाम पर जिन भौतिक सुविधाओं के आडंबर से उसे चारों ओर से घेरने का चक्रव्यूह रचा जा रहा है जो समाज के स्वास्थ्य व चरित्र विकास की दृष्टि से घातक है। क्या हुआः बदलती ऋण व्यवस्था के परिणाम-

1. डिजिटल ट्रांजेक्सन के अनिवार्य होते चलन में सरकारी बाध्यताओं के चलते बैंको की दादागीरी बढ़ हर रोज बढ़ती रही है, वे मनमाने ढंग से ट्रांजेक्सन चार्ज वसूलने में गुरेज नहीं कर रहे है और जन सेवा के ध्येय को ताक में रखते हुए बड़ी ढीटता के साथ नफाखोरी ही को अपना सर्वस्व बनाते जा रहे हैं। बैंक अपनी व्यावसायिक नैतिकता को भुलाते जा रहे हैं। बैंको के मुख्यद्वार में 
घुसते ही पूज्य गांधीजी के नाम से लिखा हुआ एक बोर्ड मिलता है कि "हमारे परिसर में आने वाला ग्राहक हमारा सम्मानीय है, हम उसकी सेवा करके उस पर कोई अहसान नहीं कर रहे अपितु वह हमें सेवा का अवसर प्रदान कर हमें अनुग्रहीत कर रहा है।" नोटबंदी व डिजिटल ट्रांजेक्सन के अनिवार्य से होने पश्चात बैंको व बैंक कर्मचारियों का रवैया बदल गया है। अब पैसा बैंक में रखना, बैंकों के मनमाने शुल्क की नीतियों का शिकार बनना तथा अमानवीय व्यवहार सहन करना इस सम्माननीय कहे जाने वाले ग्राहक की नियति बन गया है फिर भी हमारे देश के कर्णधार इस परिस्थिति, आम परेशानी से न जाने क्यों "आँख आड़े कान" किए हुए हैं। जबकि बैंक व वित्तीय संस्थाएं इन्हीं लोगों की जमाओं ऋण के रूप में विनियोजित कर दुगना-तिगुना नफा कमा रही हैं। इस प्रकार जनता का ही धन, तथा जनता के धन से ही आयोपार्जन और फिर जनता को ही ऑँख दिखाना, तरह तरह के शुल्क वसूली की निरंकुशता का प्रदर्शन और उस पर सरकारी तंत्र का उस ओर से आँख मूंद कर बैठे रहना एवं तथाकथित विकास की दुहाई देना कहाँ तक उचित माना जा सकता है? इससे ऋण के द्वारा मदद करने वाले मसीहा (बैंक और अन्य वित्तीय संस्थान) की वर्तमान भूमिका की प्रामाणिकता पर सहज ही प्रश्न खड़े हो जाते हैं।

2. अब इस ऋण व्यवस्था की फलश्रुति पर दृष्टिपात करें तो हम पाते हैं कि इस ऋण व्यवस्था और ॠणजनित सब्सीडी एवं कर्जमाफी की राजनीति से विकसित व समृद्ध किसान ही ज्यादा लाभान्वित हुए हैं। क्योंकि अधिकांश कृषिगत योजनाएं ऐसी हैं जिसमें साधन, यंत्रो अथवा अमुक टेक्नोलोजी को अपने यहाँ बसाने हेतु सब्सीडी के अतिरिक्त जितनी राशि की आवश्यकता होती है उसे जुटा पाना छोटे, सीमान्त व मध्य किसानों के बस में नहीं होता भले ही वह राशि प्रतिशत के रूप में कम प्रतीत होती हो। उदाहरण के लिए आदिवासियों के लिए 75-90 प्रतिशत सब्सीडी होने के बाद भी 25-10 प्रतिशत के रूप में 2-4 लाख की राशि का जुगाड़ बैठा पाना मुश्किल होता है। इस स्थिति में या तो प्रत्यक्ष तौर पर इस योजना का लाभ लेते ही नहीं है किन्तु कागजों में लक्ष्यांक तक पहुँचने की जुम्बिश में बड़े किसानों से मदद लेकर उसके लाभार्थी बन जाते है और ये आदिवासी महज थोड़ा सा फायदा लेकर, अथवा दबाब में आकर उस प्राप्य सुविधाओं का हवाला बड़े संभंत किसानों को दे देते हैं। कई बार ऐसा इसलिए भी होता है कि उसका संचालनीय खर्च नहीं उठा पाते। इसमें नफाकारकता की संभावनाओं के साथ साथ जोखिम का आकार भी उतना ही बड़ा होता है। महाराष्ट्र के किसानों द्वारा की गई आत्महत्याओं के पीछे छिपे तथ्य बताते हैं कि इससे कृषि उत्पादन व्यवस्था की लागतें बढ़ती हैं और प्राकृतिक प्रकोपों अथवा मानवजनित उपद्रवों का शिकार होने की स्थिति में वे इस खर्च को सहन नहीं कर पाए तथा खर्च के लिए ली गई ऋण राशि को चुकाने की संभावना नहिंवत रहने से उन्होंने आत्महत्या का मार्ग अपना लिया। 
3. एक अन्य उदहरण सामान्य वर्ग किसानों का लें जिनके लिए ग्रीनहाउस योजना प्रस्तुत की गई। इसकी एक इकाई का कुल खर्च लगभग 35 लाख है और इस पर 50 प्रतिशत सब्सीडी है अर्थात् योजना का लाभ लेने वाले के पास $17-18$ लाख की व्यवस्था आरंभिक स्तर पर होनी चाहिए तथा इसके अतिरिक्त उसके संचालकीय खर्च की व्यवस्था भी। ड्रिप सिंचाई पद्धति के संदर्भ में सरकारी सब्सीडी योजना और ऋण व्यवस्था का अवलोकन करें तो पायेंगे कि इसमें व्यापारी वर्ग को ज्यादा फायदा हो रहा है। व्यवस्था में नीतिमत्ता की कमी से काफी कुछ न खप सकने वाला माल सामान भी ठिकाने लग जाता है। आज की ग्रामीण अर्थव्यवस्था में अधिकांश किसानों की हालत इतनी समृद्ध नहीं है। बैंकिग ऋण सहायता के बाद भी उसे चुकाने के प्रश्न और जटिल हो जाते है जब किसानों को लेवी मूल्य (सरकार द्वारा जाहिर किया न्यूनतम खरीद मूल्य अथा आश्वासन मूल्य) भी नहीं मिल पाता सरकारी तंत्र की अव्यवस्थाओं एवं अनियन्त्रित व अनिश्चित बजार व्यवस्था में। कई बार हर वर्ष कहीं न कहीं किसानों का माल सड़कों पर पड़ा रहने को मजबूर होता है अथवा वे इतनी नीची कीमत पर बेचने को मजबूर होते हैं जिसमें आदानों की लागतें भी वसूल नहीं हो पाती, श्रम की कीमत को तो छोड़ ही दें। तब यह ऋण सुविधा जी का जंजाल बनती प्रतीत होती है क्योंकि इन आदानों की पूर्ति से जुड़े विभिन्न पक्षकारों के हितों पर तो कोई विशे, प्रभाव नहीं पड़ता उनका व्यवसाय तो आगे बढ़ता ही रहता है।

4. कृषिगत ऋण सुविधाऐं तो सिन्नी (मफत में मिलने वाला लाभ) जैसी हो गई हैं जो लोग एकबार खातेदार बन गए फिर भले ही वे नौकरी से जुड़े हो, खेती से उनका दूर दूर तक का सरोकार न रह गया हो, वे कम ब्याजदर के ऋण, अपरिमित ऋण राशि और कर्जमाफी का लाभ धड़ल्ले से ले रहे हैं। शैक्षणिक जागृति के चलते वे क्रेडिट कार्ड आदि का भरपूर उपयोग कर रहे हैं। पर से बैंक व सरकारों को क्या? उनका तो व्यवासायिक टर्नओवर बढ़ रहा है, सरकारी योजनाओं में हिस्सेदारी बढ़ रही है और सरकार की भी तथाकथित विकास के केनवास पर लक्ष्यांक की मुहर लग रही है। ऐसी स्थिति में सोचने की बात है कि आम जनता से कर के रूप में एकत्रित राशि का कितना भाग वास्तविक जरूरतमंद तक पहुँच सका?

5. ऋण संस्कृति के हमारी अर्थव्यवस्था के क्षेत्र में कुठाराघात किया है। ऋण मिलने से कृषि उपयोग किए जाने वाले प्रमुख आदानों खाद, बीज दवा आदि तथा साधनों का स्वरूप बदला। सजीव खेती क जगह लोग रासायनिक खेती की तरफ बढ़े। खेती की लागते बढ़ी। उत्पादन बढ़ना आज छद्म सा लगने लगा है जब बात हमारे मूलभूत संसाधन जमीन के स्वास्थ्य की हो। हरित क्रांति के बाद अभी लगभग 4 दशक ही हुए हैं सभी त्राहिमान हो उठे हैं इस व्यवस्था से इसके चलते बेशुमार रासायनिकों का उपयोग खाद और जंतुनाशक दवाओं के रूप में होने लगा। खेती की लागते बढ़ने लगीं। खेती तो बरसात का जुआ कही जाती ही थी इसलिए असफल होना, प्राकृतिक 


\section{Jain / Page 50-65}

आपदाओ का आना एक स्वाभाविक बात थी लेकिन तब किसान को कर्जा चुकाने के लिए अतिरिक्त की व्यवस्था नहीं करनी पड़ती थी। किसान जो अन्नदाता है अपनी मर्जी का मालिक कहा जाता था। बीज को सुरक्षित रखकर बाकी धान्य को उपयोग करने की परंपरा रही है ताकि आगामी फसल उगाने के लिए स्थानीय आदानों तथा श्रम के सिवाय कुछ व्यय न करना पड़े लेकिन आज खेती को व्यवसाय बना दिया। आज बीज को लेकर वह गुलाम बन गया है। वह अपना बीज खुद नहीं बना सकता। कंपनियों की एकाधिकार शक्ति व मनमानी के चलते वह हरबार नया बीज खरीदने को विविश है वह भी उन्हीं के दामों पर। अन्नपूर्णा जननी स्वरूप जमीन के पेटे से नकद फसलें प्राप्त करने के लिए पड़ापड़ी अन्धी दौड़ चालू है भले ही उसकी जरूरत उसे या स्थानीय समुदाय को न हो। इस भौतिकवादिताने जमीन के उपयोग को बदलकर रख दिया है। हरितक्रांति के पक्ष में अधिक अन्न उगाने पर जोर दिया गया था। हम विगत वर्षों का ही विश्लेषण करके देखें कि किता अन्न उत्पादन क्षेत्र नकद में परिवर्तित हो चला है और इसका प्रतिशत निरंतर बढ़ता जा रहा है। हमने बैलों को निकालकर ट्रेकटर से जुताई की कहा कि हमारे पास समय नहीं है। वहीं दूसरी ओर बेरोजगारी की दुहाई दी जाती है। जंतुनाशको के न सिर्फ हमारे उत्पादों को जहरीला बनाया अपितु आसपास के वातावरण को विषाक्त बनाकर रख दिया है। कई सारे बड़े व जटिल रोग इसी की दैन है।

6. ग्रामीण विस्तार में ऋण की स्थिति सुधरी है। किसान अपनी स्थिति सुधारने के लिए सरकारी व सहकारी बैंको से ऋण ले रहे हैं और समय पर वापस भी कर रहे हैं जिससे बैंकों का एन.पी.ए. भी नहीं बढ़ रहा। तथाकथित विकास के पुलिन्दों में इसी स्थिति का बड़ी ही सुन्दरता से चित्रण किया जा रहा है। लेखक ने गुजरात के अरवल्ली जिले के गांवों मे इसकी तहकीकात साधन सहकारी मंडलियों के संदर्भ में की तो पाया कि सत्ताधीश किसानों को ऋण लेने के लिए प्रेरित करते हैं, पुराने ऋण को ऋण को चुकाने के लिए इतनी अधिक राशि का नया ऋण भी दे देते है जिससे पुराने ऋण का बकाया चुकता हो जाता है और अतिरिक्त राशि खर्च के लिए (जरुरीऔर बिन जरूरी कार्यों पर) मिल जाती है। इससे किसान की साख सीमा भी बढ़ जाती है। है न आंकड़ों के इस खेल में सभी का फायदा! किन्तु सोचें क्या इससे किसानों की वास्तविक समृद्धि की सुनिश्चितता की जा सकती है?

7. ऋण के जरिये हर क्षेत्र में हर तरीके से सुख-समृद्धि खोजने के प्रयास और पाने क कल्पना भ्रामक ही हैं क्योंकि वर्तमान में इसके चलते उपभोगवादी संस्कृति की अति हो रही है। सुलभ ऋणों की उपलब्धता समाज में मीठा जहर फैलाने का ही काम रही है इसमें बैंकों का कारोबार खब फलफूल रहा है। किन्तु व्यक्ति को अब उसकी आय का हिसाब-किताब आने से पहले ही कल लेना होता है क्योंकि किश्तों का केंसर जो उके पीछे पड़ा होता है। ऋण लेने वाले कुछ ही समय 


\section{Jain / Page 50-65}

में न चुकापाने की स्थिति में अथवा भावी आकस्मिक जरुरतों को पूरा न कर पाने की स्थिति में अवसाद व तनाव झेलने को मजबूर हो जाते हैं। विलासी जीवनशैली की ओर बढ़ जाने से वे जाने अनजाने में जीवनशेली के विकारों को न्यौता दे बैठते हैं और इसका भान उन्हें तब होता है जब स्थिति कमोवेश उसके नियंत्रण से बाहर हो जाती है। अविवेकपूर्ण तरीके से बढ़ती इस ऋण व्यवस्था के किसानों को किसानी कार्य व वास्तविक श्रम से भी दूर सा कर दिया है, खेती को व्यवसाय में तब्दील करते किसान ने धरती माँ को बंजर बनाने में कोई करकसर नहीं रक छोड़ी, जमीन से अपेनेपन का नाता ट्ट गया, ऋण सुविधाओं के मायाजाल में कृषिभूमि पर रियायशी मकान बने, कृषिभूमि बजारीकरण की भेंट चढ़ी, धरती के परंपरागत मिजाज को ताक में रखकर लगातार नकदी पसलों का दौर चला और जो इस पर नहीं चला वही पिछड़ा साबित किया जाने लगा। जमीन में बेहिसाब झोंके जाने वाले रासायनिक खाद के जत्थे, जंतुनाशक दवाओं की भरमार ने धरती का आरोग्य तहस-नहस कर दिया हालांकि इससे मानवीय आरोग्य व पर्यावरण भी प्रभावित हुआ।

8. लोग ऋण सुविधाओं को चलते ऐसी विलासिता की वस्तुएं खरीद तो लेते है किन्तु उनके संचालन व मरम्त आदि का खर्चा भी नहीं जुटा पाते। तब उनके लिए वह वस्तु बेकार हो जाती है जिस पर उन्होंने अपनी हैसयित से अधिक खर्च कर डाला था अथवा फिर वे उचित - अनुचित का विचार किए बिना वह रास्ता अख्तियार कर लेते हैं जिसमें व्यक्ति समाज व समष्टि सभी का अहित ही होता है।

यह तो वही बात हुई न- "आ बैल मुझे मार" वास्तव में इससे जीवनशैली के विकार पनपते है। इसमें न सिर्फ व्यक्तिगत खर्च में इजाफा होता है अपितु व्यक्ति का आरोग्य खर्च भी बढ़ता है, इसके साथ साथ सरकारों का भी आरोग्य एवं कल्याणकारी खर्च बढ़ता जाता है, वास्तविक जरुरत से अधिक उत्पादन होने के कारण औद्योगिक प्रदूषण, ऊर्जा की खपत व कुदरती संसाधनों का उपयोग भी बढ़ता है जो पर्यावरणीय असंतुलन को जन्म देता है। इतना ही नहीं इसके चलते व्यक्ति कमाने की आपाधापी में इतना डूब जाता है कि वह अपना स्वाभाविक जीवन नहीं जी पाता, वह मानवीय मूल्यों के जतन की भी परवाह नहीं करता और तब इससे प्रकट होती है- सामाजिक सौहार्द व भाईचारे में कमी(लोग अपने घरों से बाहर ही नहीं निकलते), असमानता, हीनभावना व अवसाद आदि। संस्थाओ के लाभार्जन का सबब बन चुकी यह अतिरेक ऋण व्यवस्था आज व्यक्ति, समाज व समष्टि (पर्यावरण) के घातक व नासूर सिद्ध हो रही है।

9. बैंको की दोगली नीति का कहर छोटे कर्जदारों पर- आज बैंकों की स्थिति यह है कि वे बड़े कर्जदारों को ऋण देने में अपनी अधिक आतुरता बताते हैं और न चुकाने की स्थिति में न सिर्फ छूटछाट देते हैं अपितु धीरज भी बताते हैं, सैटलमेंट के नाम पर कम से कम चुकाने के प्रस्ताव 


\section{Jain / Page 50-65}

भी रखते हैं, उदारता दिखाते हैं जबकि छोटे कर्जदारों को लेकर इनका रवैया बिल्कुल ही विपरीत रहता है उनकी संपत्ति तक जप्त हो जाती है, धमकी दी जाती है तथा तरह तरह से हैरान परेशान किया जाता है। विजय माल्या जैसे करोडों के कर्जदार फारार हैं, सरकारी नीतियों के चलते आजाद व बेफिक्र घूम रहे हैं जबकि इसी देश में कर्ज को चुकाने की चिंता में किसान आत्महत्या करता है।

10.संग्रहवृत्ति को बढ़ावा अनावश्यक मूल्यवृद्धि- ऋण सुविधा के चलते व्यक्ति अपनी वास्तविक जरूरतों से अधिक खरीदी कर डालता है जिससे मांग-पूर्ति का वास्तविक संतुलन व अनुपात गड़बड़ा जाता है, क्रत्रिम अक्षत (कमी) पैदा होती है जिसका दुष्प्रभाव उस तबके पर पड़ता है जो साधन विहीन है जिसकी न वर्तमान आय की पर्याप्तता है और न ही भावी आय की कोई सुनिश्चितता।

11. स्वदेशी की जगह बाहरी लोगों का अर्थव्यवस्था पर हावी हो जाना- ऋण के इस बजार में बड़ी बड़ी कंपनियां लाभ कमाने के उद्देश्य से उतर चुकी हैं जिसमें अधिकतर बाहरी कंपनिया हैं जिन्होंने स्थानीय बजार व स्वदेशी की भावना को नेस्तनाबूत करने में कोई कसर नहीं छोड़ी है। बड़ी परियोजनाओं के लिए सरलता से ऋण उपलब्धता है जबकि कम बजट वाली उन परियोजनाओं को उस सिद्दत से प्रोत्साहन नहीं दिया जाता जिससे स्थानीय अर्थतंत्र, स्थानीय लोग मजबूत हों उनकी रचनात्मकता व उत्पादकता में मात्रात्मक व गुणात्मक रूप से निखार आए।

12. वृहद परियोजनाओं में बड़े पैमाने पर उत्पादन होने से सस्ती वस्तुएं तो उपलब्ध होती हैं किन्तु स्वदेशी हुनर व्यवस्था की कड़ी टूटती है। व्यक्ति खरीद शक्ति न होने पर भी ऋण योजनाओं के मायाजाल में सस्ती उपभोक्ता वस्तुओं को खरीदता है और ऐसा समय वह स्थानीय हुनरों के अस्तित्व व मानवीय संवेदना की परवाह न करते हुए स्वदेशी की पवित्र भावना को भुला देता है। अंग्रेजो ने उस समृद्ध भारतीय अर्थतंत्र को समाप्त करने के लिए यही नीति अपनाई थी जो अपने आप में कई प्रकार की रचनात्मक उत्पादकता व विशिष्टताओं से भरा हुई थी। निकट भविष्य इस तथ्य को नहीं नकारा जा सकता कि जो कुछ अभी शेष है वह लुप्तप्राय हो जायेगा और हमारा बचाखुचा स्वाबलंबन कमजोर पड़ जायेगा।

13.3पयोग करो और फैंक दो की संस्कृति(यूज एन्ड श्रू कल्चर) को बढ़ावा- इसकी एक बहु प्रचलित लोक पहचान चाइनीज वस्तु के रूप में बनी। निः संदेह ऋण व्यवस्था के चलते इसमें सस्ती वस्तुएं उपलब्ध हुईं किन्तु इस व्यवस्था में अधिक उत्पादन हुआ, अधिकाधिक कुदरती संसाधनों का उपयोग भी हुआ एवं अधिकाधिक प्रमाण में कचरा भी उत्पन्न हुआ। सिर्फ बजार हिस्सा व विक्रय बढ़ाने के लिए ऋण के चलते आगे बढ़े वृहद उतापदकीय प्रयास व्यक्ति व पर्यावरण विकास के लिए उपयोगी साबित नहीं हो सके। 
14. अभी क्रेडिट कार्ड जबरदस्ती दिये जा रहे हैं, टारगेट पूरे किए जा रहे हैं, जब अनावश्यक खरीदी की आदत पड़ जायेगी तो वह जरूरतें बन जायेंगी फिर कदम वापस नहीं आ सकते। अभी तो ब्याज न लेने के लुभावने ऑफर हैं, बजारीकरण की नीति के चलते क्या क्या नहीं हो सकता इसका तनिक भी अंदाज लेने वाले को नहीं होता। कर्ज लेते समय सभी को अच्छा लगता है किन्तु चुकाते समय भारी व बुरा लगता हैं। इसके चलते देनदारों की संख्या बिना किसी उचित कारण के बढ़ती चली जाती है जिससे ऋणग्रस्ता का प्रतिशत बढ़ेगा। आज बैंकिग समृद्धि के मादण्डों में बदलाव हुआ है यथा- वह बैंक बेहतर माना जाता है जिसका ऋण आवर्तन बढ़ा है इसके लिए ऋण प्रदान करने की नीतियां भी उदार बना दी गईं।

\section{उपसंहार-}

बैंको का राष्ट्रीयकरण वह कदम था जिसके माध्यम से बैंको को बाध्य किया गया कि महज नफाकारकता को ध्यान में न लें और उन क्षेत्रों तक पहुँचे जहाँ भले नफाकारकता कम हो पर महत्तम लोगों की जरूरतें पूरा होने अवकाश बढ़े। इस क्रम में ग्रामीण बैंक और क्षेत्रीय ग्रामीण बैंकिग का विस्तार हुआ। किसानों के लिए अल्पकालीन तथा दीर्घकालीन ऋणों के लिए प्रावधान किए गए, किसान क्रेडिट कार्ड वगेरे योजनाए अमल में आयीं। किन्तु परिणाम बड़े व मध्यम किसानों के हक में सर्वाधिक रहे, छूट आदि का जो भी लाभादि मिलना था उसका एक बड़ा हिस्सा समृद्ध व बड़े किसानों के हिस्से में चला गया। सहकारी बैंकिग एवं क्रेटिड व्यवस्था भी अधिक कारगर सिद्ध नहीं हुई एक ऋण को पूरा करने के लिए दूसरा बड़ा ॠण ले लेने का बढ़ता चलन- लोग ऋण के दलदल में फँसे रहे इसके लिए जिम्मेदार यह एक नया चलन ही है। व्यवस्था उतनी गलत नहीं कही जा सकती किन्तु इसके चलते समाज में अकर्मण्यता की जो मनोवृत्ति पैदा हो रही है वह इस सहायता को ऐसा केंसर का रूप दे देगी जो व्यक्ति और समाज को खोखला करके रख देगा।

पहले लोग गरीबी में ऋण लेते थे, आपत्ति-विपत्ति के समय ऋण लेते थे, सामाजिक-पारिवारिक जिम्मेदारियों को पूरा करने के लिए ऋण लेते थे, खेती के लिए ऋण लेते थे, व्यवसाय के लिए भी ऋण क जरूरत को पूरा किया जाने लगा। लोग शाहूकारों के चुंगल में रहते थे, ऋण न चुका पाने की हालत में अपनी जमीन कमाने व आजीविका के साधन से भी हाथ धोना पड़ता था। ऐसा नहीं था कि इन शाहूकारों को पता नहीं था इस अंजाम का फिर भी कास्तकारों की सम्पत्ति को देखकर उसको हड़प जाने की नीयत से उसे अधिक से अधिक कर्ज लेने के लिए उकसाते थे, कई बार ऐसे हालात स्वंय भी पैदा कर देते थे कि वह कर्ज चुका ही न सके अथवा पुराने कर्ज को चुकाने के लिए नया बड़ा कर्ज ले ले इस तरह कर्ज का दायरा बढ़ता जाये। और वह कंगाल हो जाए। 


\section{Jain / Page 50-65}

ॠण से वित्तीय संस्थाएं किसी भी अर्थव्यवस्था के संचालन में वही भूमिका निभाती हैं जो शरीर के संचालन में लहू का कार्य करती हैं। इससे उन क्षेत्रों में व्यवसाय का विकास होता है और उद्यमिता को प्रोत्साहन मिलता है किन्तु समस्या तब पैदा होती है जब आसान ऋणों की उपलब्धता के चलते लोग आधेधड़ जोखम उठाते हैं और परिणामों की परवाह नहीं करते। ऋण के इस बजार में वर्तमान व भावी आय की बात की जाती है किन्तु भावी खर्च की चर्चा नहीं होती इसलिए भ्रम पैदा होता है। ऋण अपने आप में बुरा नहीं है किन्तु समस्या है उसके विवेकपूर्ण उपयोग की, अति पर अंकुश लगाने की। ऋण बुरा न है न भला है, यह किसी के लिए जी का जंजाल है तो किसी के लिए जीव की रामावाण औषधि। भारतीय संस्कृति साहित्य में ऋण और जीवन यापन की व्यवस्थाओं को लेकर दो प्रमुख दर्शन है चार्वाक दर्शन और संयममय जीवन का दर्शन। चार्वाक दर्शन "कर्ज लो और घी पियो" की नीति का अनुसरण करता है। जो लोगों को भोग विलास वाली उपभोक्ता संस्कृति के गर्त में धकेलती है तथा समाज व भावी पीढ़ी के प्रति गैर जिम्मेदार बनाती है। उदार ऋण नीति ने चार्वाक दर्शन को बढ़ावा दिया है। जबकि संयममय जीवन की संस्कृति "तेते पांव पसारिये जेती लंबी सोर" पर बल देती है। मतलब जितनी हैसियत है उतना ही खर्चा करें, इससे उपभोक्तावादी संस्कृति के प्रसार पर अंकुश लगता है जिसमें संसाधनों का संरक्षण सुनिश्चित होता है। गांधीजी भी ता उम्र अपने जीवन में संयममय जीवनशैली के अनुकरण पर जोर देते रहे। किन्तु समाज के वर्तमान विकास एवं उद्यमिता विकास के अवसर खड़े करने हेतु ऋण की आवश्यकता को नकारा नहीं जा सकता। क्या ऋण से रोजगार के अवसर पनपते हैं? यदि इस प्रश्न का उत्तर हां में है तो हमें विचार करना होगा कि ऋण किस सीमा तक हों। अन्यथा सीमा के लांघने पर एक बड़े समुदाय को स्वाबलंबी बनाने वाली, रोजगार सुनिश्चित करने वाली विकन्द्रित उत्पादन व्यवस्था बिखरती है, बड़े पैमाने पर उत्पादन व मशीनीकरण की ओर उन्मुख होती है जिसके केन्द्र से धीरे धीरे मानव अदश्य होता होता चला जाता है और सभी कुछ स्वचलित होता चला जाता है फिर मानवदिन, रोजगारी का सपना केन्द्रीकृत अर्थव्यवस्था की भेंट चढ़ जाता है। अब जरा सामाजिक क्षेत्र में भी ऋण के दीर्घगामी प्रभाव को देखें- उच्च व बेहतर शिक्षा के लिए शिक्षण ऋण सुविधा उपलब्ध है। इसने तो शिक्षा क्षेत्र को व्यवसाय बनाकर रख दिया है।

गांधीजी ने संयममय जीवन के अभिगम को अपनाने की आवश्यकता पर इसीलिए बल दिया एक तरफ इससे अतिरेक उत्पादन में लगने वाले साधन-संसाधनों का विनाश होने से बचेगा, पर्यावरण संतुलित रह सकेगा तथा लोगों रात-दिन कमाने तथा ऋण भुगतान करने की तानाव से मुक्ति मिल सकेगी जो उन्हें शांति व सुखमय जीवन जीने के लिए प्रेरित करेगी क्योंकि ऋण ऐसी प्यास है कि जो बुझने का नाम ही नहीं लेती, अवास्तविक सुख की ओर आकर्षित कर अंततः जोखिम की तरफ ढकेल देती है। 


\section{संदर्भ-}

1. मो.क. गांधी- "हिन्द स्वराज", नवजीवन प्रकाशन, अहमदाबाद

2. बिनोवा भावे- "लफंगा पैसानुं का अनर्थकारण" (गुजराती), यज्ञ प्रकाशन, वडोदरा

3. जैन (डॉ.) लोकेश

(अ) “Entrepreneurial spirit among SHG towards women empowerment (Study with reference to Pundhara village in Manasa taluka of Gandhinagar district in Gujarat)" paper presented in Tenth Biennial Conference on Entrepreneurship(2013), EDI, Ahmedabd. And published by Book well, New-Delhi, ISBN- 978-80574-46-02 (vol-1), page no. 534-548.

(आ) "Role of Traditional Wisdom to manage the lifestyle disorder for sustainable human development" published in edited book Relevance of Gandhian Thoughts in search of Sustainable Dimension in Family and Community Science, (2014), Garg Publications, Ahmedabad, ISBN:979-82- 320-2535-5

(इ) "Inclusive Economic Growth and Sustainable Rural Development in Tribal Area of Gujarat: Gandhian View of Development (A Study of Dang district in Gujarat)" paper presented in UGC sopnsored National Seminar on Inclusive Growth: An Opportunity with Challenges organized by department of Economics, S.R.K. (P.G.) college, Firozabad (U.P.) and published in Proceeding Book ISBN 978-935254-260-4,

(ई) ग्रामीण लोकोनी बदलाती उपभोगवृत्ति अने सम्पोषित विकास पर असर (गुजराती) के.सी.जी. ई-जर्नल ऑफ सोशल साइंस, अंक-21, अप्रल-मई 2016 ISSN- 2279-0241 पेज- 1- 11.

\section{डॉ. लोकेश जैन \\ प्राध्यापक, ग्रामीण प्रबंध विभाग, प्रबंधन एवं प्रोद्योगिकी संकाय गूजरात विद्यापीठ, ग्रामीण परिसर-रांधेजा-गांधीनगर (गुजरात) \\ E-mail: lokeshcsrm@yahoo.co.in}

